\title{
Investigation of Ferritin Desorption from Gold Initiated by In Situ pH-Change
}

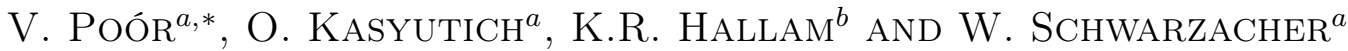 \\ ${ }^{a}$ Department of Physics, University of Bristol, Bristol, UK \\ ${ }^{b}$ Interface Analysis Centre, University of Bristol, Bristol, UK
}

\begin{abstract}
One of the main questions regarding protein adsorption is about the reversibility of the adsorption process. To get a deeper understanding of this, adsorption of ferritin on Au was studied by quartz crystal microbalance and the $\mathrm{pH}$ of the buffer was changed in situ between two values that favour adsorption by different amounts. We found that although some ferritin desorbs from $\mathrm{Au}$, the desorption is incomplete. When the desorption reached a constant value, we returned to the original conditions and investigated the readsorption. Our experiments show that the adsorption of ferritin onto $\mathrm{Au}$ is a partly reversible process. We found that for different initial ferritin coverages the proportion of ferritin that had been subsequently desorbed was approximately constant.
\end{abstract}

PACS numbers: 81.07.Nb, 87.14.E-, 87.15.R-

\section{Introduction}

Protein adsorption on surfaces is a well-studied phenomenon, as protein-surface interactions are present in life and are useful for technological applications [1-7]. At the same time they are sometimes undesirable, as they can lead to formation of biofilms or cause thrombosis [5].

Therefore the reversibility of protein adsorption onto a wide range of surfaces is a broadly studied subject. Various theories were developed to analyse these processes and there are several experimental reports [6-9]. However, there exists no entirely satisfactory, comprehensive theory.

To help the development of further theories and to gain deeper understanding of the protein adsorption onto surfaces, we decided to monitor in situ the adsorption and desorption processes of a selected protein induced by the change of $\mathrm{pH}$. The protein needs to be stable in the investigated $\mathrm{pH}$-range, and be easy to describe and model.

We have chosen native horse spleen ferritin as a useful tool for our investigation of protein adsorption on Au surfaces. Ferritin is a quasispherical protein, whose function is to store iron in living organisms. The inorganic core of ferritin has a diameter of about $8 \mathrm{~nm}$ and can contain up to $4500 \mathrm{Fe}$ (III) ions [10], while the external diameter of the protein is $12 \mathrm{~nm}$. Ferritin is a good choice for gravimetric investigation by quartz crystal microbalance (QCM) [1-3] as the inorganic iron-containing core has a much higher density than a typical protein without inorganic component, which means that the signal-to-noise ratio on the QCM is enhanced, giving more accurate mea-

* corresponding author; e-mail: veronika.poor@bris.ac.uk surements of the adsorbed amount. Even though not every ferritin core has the same mass, when averaging over a large surface the differences are negligible.

In their previous work, Caruso et al. [1] showed that the adsorption of ferritin is dependent on concentration in the range of $1-20 \mu \mathrm{g} \mathrm{ml}^{-1}$, while Hemmersam et al. [2] showed that the adsorption of ferritin is also dependent on the surface properties such as the isoelectric point and the $\mathrm{pH}$.

In this paper we show for the first time that it is possible to alternate between adsorption and desorption of ferritin on $\mathrm{Au}$ by changing the $\mathrm{pH}$ of the solution in situ. We also show that although there is some degree of reversibility in the adsorption of ferritin, it is far from being a completely reversible process. We further present results of how ferritin desorption depends on the amount previously adsorbed.

\section{Materials and methods}

The horse spleen ferritin (Sigma-Aldrich, in 0.150 M $\mathrm{NaCl}$ ) was used as received. (Tests carried out with ferritin that we have purified further did not show qualitative differences to the as-received material.) The buffer solution contained $5 \mathrm{mM}$ HEPES (Fisher Scientific, $99 \%+$ ), $5 \mathrm{mM}$ acetic acid (Aldrich, 99.7\%+) and $100 \mathrm{mM} \mathrm{KCl}$ (Sigma-Aldrich, 99-100.5\%), dissolved in MilliQ water (resistivity $18.2 \mathrm{M} \Omega \mathrm{cm}$ ). The $\mathrm{pH}$ of the buffer was changed by addition of $\mathrm{KOH}$ and $\mathrm{HCl}$.

The QCM measures the frequency shift caused by the adsorbed mass on an oscillating piezoelectric crystal. In the case of small mass changes the Sauerbrey equation [11] applies 
$\Delta f=C_{f} \Delta m$,

where $f(5 \mathrm{MHz})$ is the frequency of the crystal, $C_{f}$ is a constant for a given quartz crystal and $\Delta m$ is the adsorbed mass. The Sauerbrey equation is deduced for oscillation in a gas environment, therefore in our case viscoelastic effects from the solvent and the adsorbed layer complicate the situation. Based on the results of Caruso et al. [1] the theoretical value of $C_{f}$ for in-air measurements has to be multiplied by $\approx 2.3$ when applied to ferritin adsorbed from liquid. Due to the method used by Caruso et al. [1], this estimate should probably be considered as a maximum value.

The adsorption data were measured using a Stanford Research Systems QCM 200, and quartz crystals (2.54 cm diameter, AT-cut Stanford Research Systems) coated with sputtered Au.

The Au-coated crystals were cleaned using Piranha solution ( 1 part $30 \% \mathrm{H}_{2} \mathrm{O}_{2}$ and 3 parts $96 \% \mathrm{H}_{2} \mathrm{SO}_{4}$ ) for $20 \mathrm{~min}$, then rinsed thoroughly with MilliQ water [1]. The cleanliness of the crystal was ensured by checking the frequency in air.

The solution was stirred (300 rpm) and the $\mathrm{pH}$ was monitored in situ, using a PHM210 standard pH meter (MeterLab, Radioanalytical, Copenhagen). The measurements were conducted at room temperature.

\section{Results}

We made various measurements to find out more about the adsorption and desorption of ferritin onto/from Au. The reproducibility of our results was around $20 \%$, which is comparable to the reproducibility reported by Ferapontova et al. [4].

The charge on the ferritin surface can be varied by a change of $\mathrm{pH}$. The isoelectric point $(\mathrm{pH}(\mathrm{I}))$ of the ferritin being around $4.6[12]$ implies that the ferritin is negatively charged above this $\mathrm{pH}$. If the $\mathrm{pH}$ of the buffer is 5 , there is a weak electrostatic repulsion between the ferritin molecules themselves - which increases considerably if the $\mathrm{pH}$ is changed to higher values making the surface charge of ferritin more negative [2]. An IP cannot be stated for $\mathrm{Au}$, but previous research suggests that the adsorption of ferritin on $\mathrm{Au}$ reaches its maximum at $\mathrm{pH} 5$ and then diminishes as the $\mathrm{pH}$ of the solution is increased [2]. In our case we changed the $\mathrm{pH}$ to 7.5 in situ, to get a deeper insight into the reversibility of ferritin adsorption on $\mathrm{Au}$.

Figure 1 shows how the system reacts to a $\mathrm{pH}$ change in situ. The ferritin was adsorbed at $\mathrm{pH} 5.0 \pm 0.1$ for $10 \mathrm{~min}$, after which the $\mathrm{pH}$ was changed to $7.5 \pm 0.1$ by the injection of $5 \mathrm{M} \mathrm{KOH}$ solution, to initiate desorption. After 15 min at $\mathrm{pH} 7.5 \pm 0.1$ the $\mathrm{pH}$ of the solution was reset to $5.0 \pm 0.1$ by the injection of the appropriate amount of $20 \% \mathrm{HCl}$ solution to favour adsorption again. As the solution was stirred during all of our experiments, homogeneity was reached after seconds. This experiment demonstrates the feasibility of alternating between adsorption and desorption of ferritin on $\mathrm{Au}$ by changing the $\mathrm{pH}$ of the solution in situ.

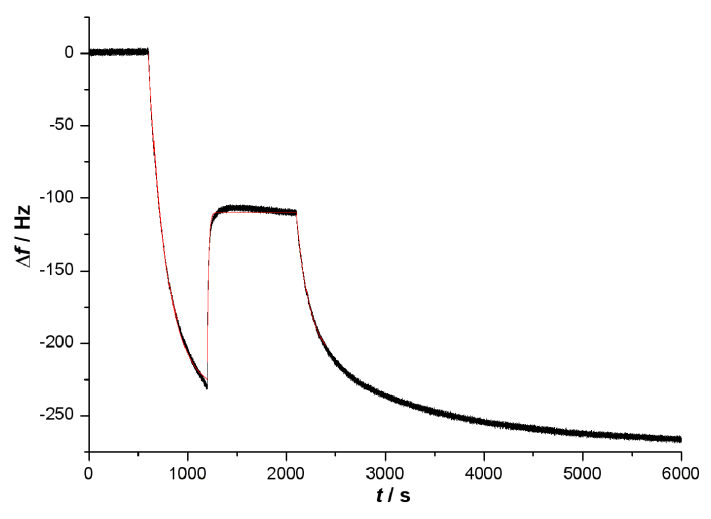

Fig. 1. pH-dependent adsorption and desorption of ferritin. Ferritin (final concentration $100 \mu \mathrm{g} \mathrm{ml}^{-1}$ ) was added at $t=600 \mathrm{~s}$. The adsorption started at the moment of addition. At $t=1200 \mathrm{~s}$ the $\mathrm{pH}$ of the solution was changed to $7.5 \pm 0.1$ from the initial value of $5.0 \pm 0.1$, and this induced immediate desorption. At $t=2100 \mathrm{~s}$ the $\mathrm{pH}$ of the buffer was returned to $5.0 \pm 0.1$, when readsorption occurs. Exponential curves (for further details see in the text) were fitted to the adsorption and desorption curves. In our raw data there is a small linear term which we attribute to drift, and which is constant and independent of $\mathrm{pH}$. We subtracted this term prior to plotting.

To see whether the frequency change reached at the higher $\mathrm{pH}$ is an absolute value, we measured the desorption after various adsorption times (2, 5 and $10 \mathrm{~min})$. Different adsorption times correspond to different amounts of ferritin initially adsorbed. Figure 2 shows that the steady-state value reached at $\mathrm{pH} 7.5 \pm 0.1$ is indeed dependent on the initial adsorption time or the adsorbed amount. The data show that under our experimental conditions the proportion of initially adsorbed ferritin that desorbs is approximately fixed at around $50 \pm 5 \%$.

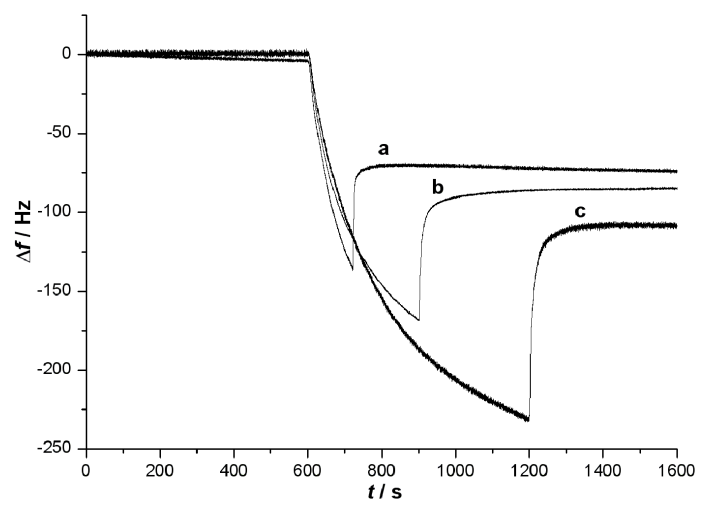

Fig. 2. Time dependence of ferritin desorption. Ferritin (final concentration $100 \mu \mathrm{g} \mathrm{ml}^{-1}$ ) was added at $t=600 \mathrm{~s}$. After letting ferritin adsorb for: (a) $120 \mathrm{~s}$, (b) $300 \mathrm{~s}$, and (c) $600 \mathrm{~s}$, desorption was initiated by changing the $\mathrm{pH}$ from $5.0 \pm 0.1$ to $7.5 \pm 0.1$. 


\section{Discussion}

\subsection{The kinetics of adsorption}

Providing the fitting interval is not too long, the time-dependence of the frequency change can be well described by a simple exponential approach to saturation

$$
\Delta f=l\left[1-\exp \left(-\Delta t / t_{\mathrm{ads}}\right)\right],
$$

where $\Delta f[\mathrm{~Hz}]$ is the frequency change of the oscillating quartz crystal, $\Delta t[\mathrm{~s}]$ is the adsorption time, $l$ is the saturation frequency change, which is a measure of the maximum amount of ferritin that may be adsorbed, and $t_{\text {ads }}$ is a time constant for adsorption. $t_{\text {ads }}$ will likely depend on the protein concentration close to the crystal surface.

For longer intervals (2) breaks down, which is perhaps not surprising as it is based on a very simple model of irreversible adsorption. The effective time constant for adsorption $t_{\mathrm{ads}}$ is smaller for readsorption $(143 \pm 14 \mathrm{~s})$ than for the initial adsorption $(186 \pm 18 \mathrm{~s})$, perhaps because in the former case the local concentration is higher due to some ferritin that was desorbed remaining in the vicinity of the substrate.

\subsection{Desorption induced by $p H$ change}

To see whether the adsorption of ferritin on $\mathrm{Au}$ is a reversible process, the $\mathrm{pH}$ and hence the charge on the protein was changed in situ. The fact that changing the $\mathrm{pH}$ initiates desorption already implies that there is some reversibility in the system. The desorption is not complete, which could be caused by a change in state of some of the adsorbed protein. Our result that $50 \pm 5 \%$ of the adsorbed protein is desorbed independently of the initial adsorption time/adsorbed amount suggests that the proportion of protein that adsorbs reversibly is approximately the same at all stages of the process. Further experiments are needed to determine whether protein that is initially adsorbed reversibly can transform to an irreversibly adsorbed form on longer timescales.

\subsection{The kinetics of desorption}

The kinetics of desorption can also be described by an exponential curve, where the limit for large $t$ is dependent on the preceding adsorption time/previously adsorbed amount. The equation of the fitted curve is

$$
\Delta f=r\left[\exp \left(-\Delta t / t_{\mathrm{des}}\right)\right]+i
$$

where $t_{\text {des }}$ is a time constant for desorption. In this equation $r$ is proportional to the reversibly adsorbed protein amount and $i$ to the irreversibly adsorbed amount. In the case of desorption after 10 minutes adsorption time, $r$ equals $-101 \pm 10 \mathrm{~Hz}, t_{\text {des }}=22 \pm 10 \mathrm{~s}$ and $i=-110 \pm 11 \mathrm{~Hz}$. In the case of initial adsorption for $2 \mathrm{~min} i$ equals $-72 \pm 7 \mathrm{~Hz}$; in the case of $5 \mathrm{~min}$ $i=-86 \pm 9 \mathrm{~Hz}$.

If we compare $t_{\mathrm{des}}$ and $t_{\mathrm{ads}}$, we see that $t_{\mathrm{des}}$ is an order of magnitude smaller than $t_{\mathrm{ads}}$, which means that the kinetics of desorption is faster than those of the adsorption. This result is consistent with the adsorption being limited by the near-surface concentration while in the case of desorption this is not a major factor.

\section{Conclusions}

We have shown that the desorption of ferritin from $\mathrm{Au}$ can be induced by changing the $\mathrm{pH}$ of the buffer in situ. By comparing rates of adsorption and desorption we find evidence that the adsorption of ferritin is limited by the near-surface concentration of protein in our system. We do not observe significant dependence of the proportion of ferritin that desorbs on the initially adsorbed amount. Although at the atomic and molecular level, protein adsorption is a highly complex process, our results show that a simple model appears sufficient to describe the observed kinetics. Similarly simple pictures have been used to interpret the results of previous studies $[1,2]$.

\section{Acknowledgments}

This project is supported by the Marie Curie Early Stage Training Programme (MEST-CT-2005-020828) MISSION of the European Commission. The data from the QCM were recorded by a modified version of a LabView program originally written by Jonathan Velleuer.

\section{References}

[1] F. Caruso, et al., J. Colloid Interface Sci. 186, 129 (1997).

[2] A.G. Hemmersam, et al., J. Phys. Chem. C 1124180 (2008).

[3] K. Uto, et al., J. Mater. Chem. 183876 (2008).

[4] E. Ferapontova, et al., Bioelectrochemistry 55127 (2002).

[5] M.A. Cole, et al., Biomaterials 301827 (2009).

[6] P.R. Tassel, et al., J. Chem. Phys. 106761 (1997).

[7] M.J. Mura-Galelli, et al., Biochemistry $\mathbf{8 8} 5557$ (1991).

[8] H. Liu, et al., Colloids Surf. B 535 (1995).

[9] J. Talbot, Adsorption 289 (1996).

[10] G. Thomas, Medicinal Chemistry, Wiley, New York 2000.

[11] G. Sauerbrey, Z. Phys. 155206 (1959).

[12] S.T. Silk, et al., J. Biol. Chem. 2516963 (1976). 